\title{
Investigation on a choice of stator slot skew angle in brushless PM machines
}

\author{
M. Jagiela • E. A. Mendrela • P. Gottipati
}

Received: 22 April 2010 / Accepted: 12 September 2012 / Published online: 2 October 2012

(C) The Author(s) 2012. This article is published with open access at Springerlink.com

\begin{abstract}
The paper investigates the effects of stator slot skewing in a permanent magnet brushless DC motor. A simple analytic formula for calculation of the best angle of stack skew, which leads to nearly total reduction of the cogging torque, is developed. The skew angle obtained from this formula is different to that used by the designers of PM brushless motors. The analysis is carried out for a fractional horsepower brushless permanent magnet motor with the surface-mounted magnets using a time-stepping, multi mesh-slice finite element model, to assess the impact of this change. The steady-state characteristics and core losses are analyzed quantitatively using the elaborated numerical model. It is shown that smaller skew angles obtained from the formula lead to noticeable rise in motor overall efficiency and decrease of the core loss. The possibility of accomplishment of the desired effect of skew in a real machine is also a subject of discussion.
\end{abstract}

Keywords Permanent magnet brushless DC motors .

Slot skew $\cdot$ Core loss $\cdot$ Electromagnetic torque .

Finite element analysis

M. Jagiela ( $\square)$

Department of Electrical Engineering, Automatic Control

and Computer Science, Opole University of Technology,

ul. Proszkowska 76, 45-758 Opole, Poland

e-mail: m.jagiela@po.opole.pl

\section{E. A. Mendrela $\cdot$ P. Gottipati}

Electrical and Computer Engineering Department,

Louisiana State University, Baton Rouge,

LA 70803, USA

e-mail: ermen@ece.1su.edu

P. Gottipati

e-mail:pgotti1@1su.edu

\section{List of symbols}

$\mathrm{A}$

$d$

$f$

$H_{c u}$

$i_{u}$

$v_{\mathrm{S}}$

$\ell$

$N$

$n_{\mathrm{S}}$

$n$

$n_{\mathrm{t}}$

$L_{e u}$

$\boldsymbol{L}$

$p$

$q$

$Q$

$Q_{\text {s }}$

$R_{u}$

$\boldsymbol{R}$

$S_{n}$

$T$

$v_{u}$

$\alpha$

$\alpha_{s k}$

$\Delta t$

$\lambda_{u}$

$v$

$\varphi$

$\tau$ $z$ th component of magnetic vector potential

Thickness of electrical sheet

Frequency

Components of coercive magnetic field vector, $u=x, y$

Phase current, $u=a, b, c$

Vector of branch voltage sources

Length

Finite element shape function

Number of slices (submodels) of multi mesh-

slice model

Time-step index

Number of turns

Inductance of phase end-winding, $u=a, b, c$

Matrix of branch inductances

Number of pole-pairs

Ratio of pole-width to pole-pitch

Least number of slots in a doubly slotted machine

Number of coils per phase of distributed winding

Resistance of phase-belt, $u=a, b, c$

Matrix of branch resistances

Cross-sectional area of $n$th coil

Time-period

Supply voltage of $u$ th phase-belt, $u=a, b, c$

Mechanical angle

Skew angle

Time-step length

Phase-belt flux linkage, $u=a, b, c$

Magnetic reluctivity

Nodal value of magnetic vector potential

Angular width of one slot-pitch 
$\sigma \quad$ Electric conductivity

$\rho \quad$ Mass density

$\eta_{n} \quad$ Auxiliary coefficient equals 1 if current sense in $n$th coil is the same with $z$ direction and -1 , otherwise

$\eta_{u} \quad$ Auxiliary coefficient equals 1 if coil belongs to $u$ th phase-belt and 0 , otherwise

$\Omega \quad$ Region of analysis

$\nabla \quad(\partial / \partial x, \partial / \partial y, \partial / \partial z)$

\section{Introduction}

There are vast research works dealing with the cogging torque reduction issues in the permanent magnet machines, e.g. [1-7]. Although much has been done in this area to date, newer publications show that it is still of significant concern to the designers of permanent magnet machines [7-11]. Among many approaches developed to cope with this problem, the skew of the stator stack sheet pack is the most natural and perhaps, the simplest method of preventing the machine from generating the reluctance-type torques to apply at the design stage [1,3,7-11]. In permanent magnet machines, the major task of skew is to get rid of the cogging torque. In all machines, the angle of skew should be selected among values of mechanical angles between 0 and $2 \pi / Q$, where $Q$ stands for the least number of slots on either stator or rotor side. A basic role of skew should be the cancellation of the most influential slot harmonic of the magnetic field which interacts with the fundamental stator mmf harmonic to produce the reluctance-type torque. An appropriate choice for the angle of skew can be made only after detailed investigation on the magnetic field distribution in the machine airgap, because a principle of generation of the electromagnetic torque is complex [7].

In this work, the authors show a different point of view on the application of the stack skew in the permanent magnet machines. The difference is in the value of the skew angle used. We developed a simple formula for best skew angle which is different to that used by the majority of designers $[1,3,5,7]$. This formula results from the analysis of the distribution of the magnetic field harmonics in the motor air-gap, like the other methods, but it gives best angle of skew for the particular motor, and not the minimum one like the other approaches. In the conventional methods, the starting point of the considerations is a skew factor which is a component of winding factor $[10,11]$. In the simplest manner, the influence of skew on $j$ th harmonic of the magnetic flux density is considered the same with an averaging filter:

$B_{s k j}=\frac{1}{\alpha_{s k}} \int_{-0.5 \alpha_{\mathrm{sk}}}^{+0.5 \alpha_{s k}} B_{n s j} \cos (j p \alpha) \mathrm{d} \alpha$, where $B_{n s}$ is a magnitude of magnetic flux density in the machine without skew, and $B_{s k}$ is a magnitude of flux density in the same machine with skew. The skew factor is:

$k_{s k j}=\frac{B_{s k j}}{B_{n s j}}=\frac{2 \sin \frac{j p \alpha_{s k}}{2}}{j p \alpha_{s k}}$

The minimum angle of skew is determined from (2) as to provide zero value of skew factor for the most influential harmonic order. The most common choice for skew angle is $\alpha_{s k}=\tau$. If this is the case, the most suppressed harmonic would be of order $Q / p$, because from (2):

$\alpha_{s k}=\frac{2 k \pi}{Q}, \quad k=1,2, \ldots n$

for $k=1$ is understood as the minimum value, equal to $\tau$.

In the above formula, the choice of most influential harmonic number is suggested by the mathematical formula without physical considerations on the magnetic field distribution in the motor air-gap. It can be shown that the best angle of skew can be even smaller than that suggested by (3) when the reference quantity of the considerations is the electromagnetic torque not the magnetic flux density. The skew angle given by (3) did not result in total reduction of the cogging torque. A much better value of the skew angle can be deduced in the following way.

Assume a permanent magnet machine at standstill with the number of poles $2 p$ and the number of slots $Q$. For simplicity, also assume $Q / p$ being integer. The order of the fundamental harmonic of the cogging torque in all permanent magnet machines (excluding the fractional-slot machines) is the least common multiple of 2 and number of slots divided by number of pole-pairs. In such a case, an obvious choice for skew angle would be angular width of one slot pitch, as it is done in (3). On the other hand, it is well known that the principle of generation of the electromagnetic torque is based on a cross product between all terms of Fourier series expansion of the distributions of radial $\left(B_{\mathrm{r}}\right)$ and tangential $\left(B_{\psi}\right)$ components of magnetic flux density. This is because an instantaneous value of the electromagnetic torque is in proportion to the product $B_{\mathrm{r}} \cdot B_{\psi}$ [7]. This analytical operation results in an infinite sum of products of trigonometric functions. Each such product can be further expanded into a sum of two trigonometric functions of the sum and the difference between their arguments.

Most of the cogging torque produced in permanent magnet machines comes from an interaction of the fundamental flux density harmonic, due to magnets, with the most influential harmonic due to slotting [7]. It is well-known that in machines having integer $Q / p$ all slot harmonic ordinals are contained in series: $h \cdot(Q / p)+1$, with $h= \pm 1, \pm 2, \ldots$ An order of the fundamental harmonic of the cogging torque is known. It is always equal to $(Q / p)$ or, more generally, 
Table 1 Relative skew angles calculated from (4) referred to slot-pitch width (integer-slot machines)

\begin{tabular}{lllllllll}
\hline$p$ & $Q$ & & & & & & & \\
\cline { 2 - 8 } & 12 & 18 & 24 & 30 & 36 & 42 & 48 & 54 \\
\hline 1 & 0.923 & 0.947 & 0.960 & 0.967 & 0.973 & 0.976 & 0.979 & 0.981 \\
2 & 0.857 & $\times$ & 0.923 & $\times$ & 0.947 & $\times$ & 0.960 & $\times$ \\
3 & $\times$ & 0.857 & $\times$ & $\times$ & 0.923 & $\times$ & $\times$ & $\times$ \\
4 & $\times$ & $\times$ & 0.857 & $\times$ & $\times$ & $\times$ & 0.923 & $\times$ \\
5 & $\times$ & $\times$ & $\times$ & 0.857 & $\times$ & $\times$ & $\times$ & $\times$ \\
6 & $\times$ & 0.428 & $\times$ & $\times$ & 0.857 & $\times$ & $\times$ & $\times$ \\
7 & $\times$ & $\times$ & $\times$ & $\times$ & $\times$ & 0.857 & $\times$ & $\times$ \\
8 & $\times$ & $\times$ & 0.428 & $\times$ & $\times$ & $\times$ & 0.857 & $\times$ \\
9 & $\times$ & $\times$ & $\times$ & $\times$ & $\times$ & $\times$ & $\times$ & 0.857 \\
10 & $\times$ & $\times$ & $\times$ & 0.428 & $\times$ & $\times$ & $\times$ & $\times$ \\
\hline
\end{tabular}

$\operatorname{lcm}(2, Q / p)$. Following this principle it can be deduced that it results from interaction between fundamental harmonic of the magnetic field and one of the harmonics having ordinals $Q / p+1(\mathrm{~h}=1)$ or $-Q / p+1(\mathrm{~h}=-1)$. Consequently, to calculate best the angle of skew for the machine with integerslot type winding, one can use one of the following expressions:

$\alpha_{s k}=\frac{2 \pi}{p \cdot(\operatorname{lcm}(2, Q / p)+1)}$

For the case when harmonic $Q / p+1$ is more influential than harmonic $-Q / p+1$, and:

$\alpha_{s k}=\frac{2 \pi}{p \cdot(\operatorname{lcm}(2, Q / p)-1)}$

otherwise. It is much more advantageous to use the skew angle calculated from (4) that resulted from (5), not only due to higher skew factor involved. Such a choice is also motivated by the fact that in the surface-mounted machines besides slot harmonics having ordinal $-Q / p+1$ there is a harmonics due to permanent magnets having the same absolute ordinal, but a reverse sign. In such a situation, the negative slot harmonic will be weakened, whilst the positive one will be strengthened by the field due to permanent magnets. Consequently, Eq. (4) becomes the most appropriate choice for machines with the surface-mounted magnets having integer $Q / p$.

Table 1 summarizes values of skew angles given by (4) for the most commonly used arrangements of rotor pole-pairs and stator slot numbers.

From the results summarized in Table 1, it can be observed that with increasing number of slots the angle of skew approaches the angular width of one slot-pitch, which is the most commonly used by designers. Skewing as a method of cogging torque reduction is usually applied to machines with distributed windings where the unity coil-span and distribution factor can be achieved relatively easily rather than in those with concentrated windings. This is due to the fundamental coil-span factor being less than unity and to the value of fundamental skew factor which has to be significantly smaller.

The skew affects all harmonics of the magnetic flux density, not only the slot harmonics, and the adequate analysis method must be involved to assess the results after application of skew angle resulted from (4). The skew factors resulted from this formula are slightly higher than those resulted from (3), and consequently it is worth investigating how application of new skew angle values influences the overall performance of machine as it may lead to costeffective solutions. To characterize these effects quantitatively, the authors develop a quasi-3D multi mesh-slice finite element model [12]. An investigation is presented including the model development and the analysis of machine operation under no-load and full-load conditions. Additionally, the analysis of core loss is presented. The results are partially backed by measurements carried out on the laboratory test stand for a prototype outer rotor brushless DC motor, designed for a gearless drive of an electrically powered wheelchair.

\section{Laboratory machine}

Figure 1a-c depicts the supply system, a stator, and a winding configuration for the motor. The technical specifications are summarized in Table 2. The motor is fed from $24 \mathrm{~V}$ batteries via the two-level voltage wave inverter and operates in 120 electrical degree mode of commutation with discrete position sensors. 


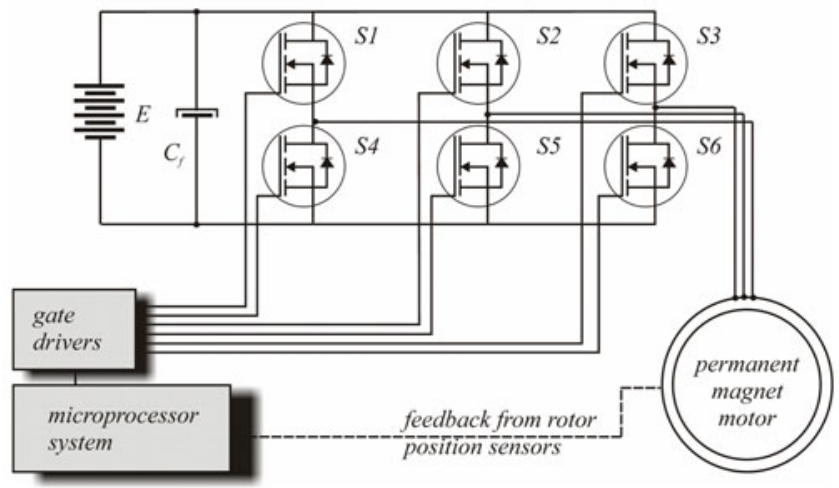

(a)

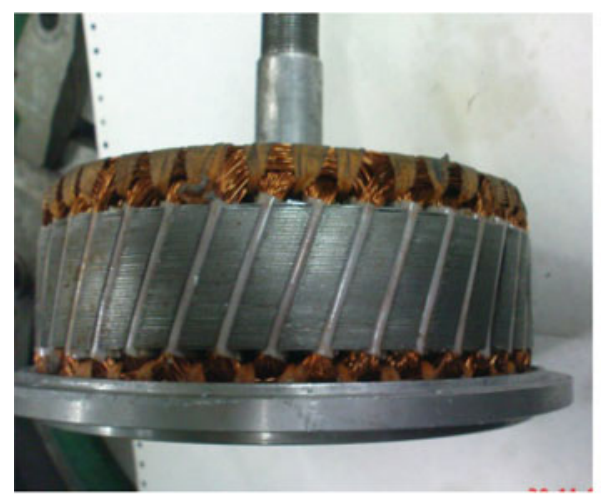

(b)

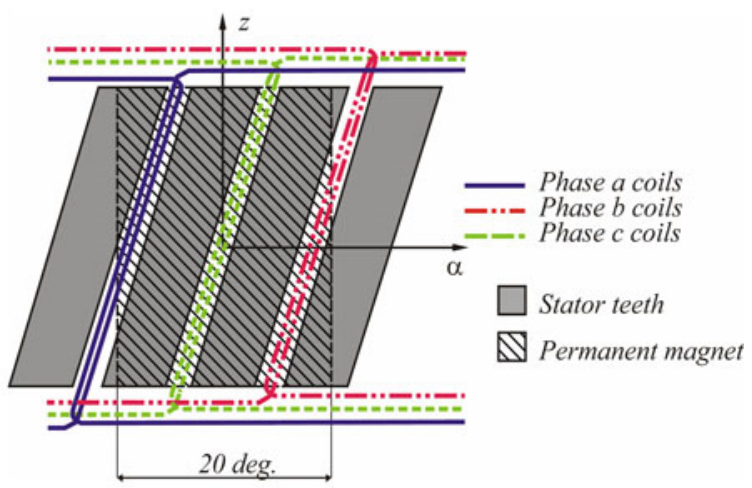

(c)

Fig. 1 Permanent magnet brushless DC motor supply system (a), skewed stator (b), relative position of magnet versus stator teeth (c)

\section{Motor model for performance analysis}

A quasi-3D numerical model for magnetic computation assumes uniform magnetic field distribution along with single two-dimensional submodel (slice) in $z$ direction [12]. The whole model is composed of $n_{\mathrm{s}}$ such slices. In case of an outer-rotor configuration, the $q$ th submodel (see Fig. 2) contains the geometry with stator geometry moved to angular position:

$\alpha_{s k q}=\left(\frac{q-1}{n_{\mathrm{s}}-1}-\frac{1}{2}\right) \alpha_{s k}$

A distribution of two-dimensional magnetic field over $q$ th submodel is governed by the Ampére's law [6,7]:

$$
\left(v \frac{\partial^{2} A}{\partial x^{2}}+v \frac{\partial^{2} A}{\partial y^{2}}\right)_{q}=-\sum_{u} \sum_{n=1}^{p Q_{s}} \frac{n_{\mathrm{t}} \eta_{u} \eta_{n} i_{u}}{S_{n}}+\left(\nabla \times \mathbf{H}_{c}\right)_{z_{q}}
$$

where subscript $z$ denotes $z$ th component of vector product. The equation that describes connection of electric circuits associated with coils of single phase of the motor is:
Table 2 Specifications of the prototype motor
Outer/inner rotor diameter

Outer/inner stator diameter

Length

Rotor configuration

Number of pole-pairs

Number of stator slots

Angle of stator skew

Winding

Nominal voltage, power

Nominal speed

\section{$194 / 170 \mathrm{~mm} / \mathrm{mm}$ \\ $168 / 110 \mathrm{~mm} / \mathrm{mm}$ \\ $40 \mathrm{~mm}$}

Outer with surface-mounted magnets

7

42

One slot-pitch

Double layer, full pitch, 18 turns per slot

$24 \mathrm{~V}, 200 \mathrm{~W}$

$170 \mathrm{rpm}$
$v_{u}=R_{u} i_{u}+L_{e u} \frac{\mathrm{d} i_{u}}{\mathrm{~d} t}+\sum_{q=1}^{n_{\mathrm{s}}}\left(\frac{\mathrm{d} \lambda_{u}}{\mathrm{~d} t}\right)_{q}$

A distributed winding without parallel paths is assumed. The flux linkages in (8a) are evaluated from: 
Fig. 2 Skewed stator of an outer rotor permanent magnet motor (a) represented by means of multi-slice model (b)

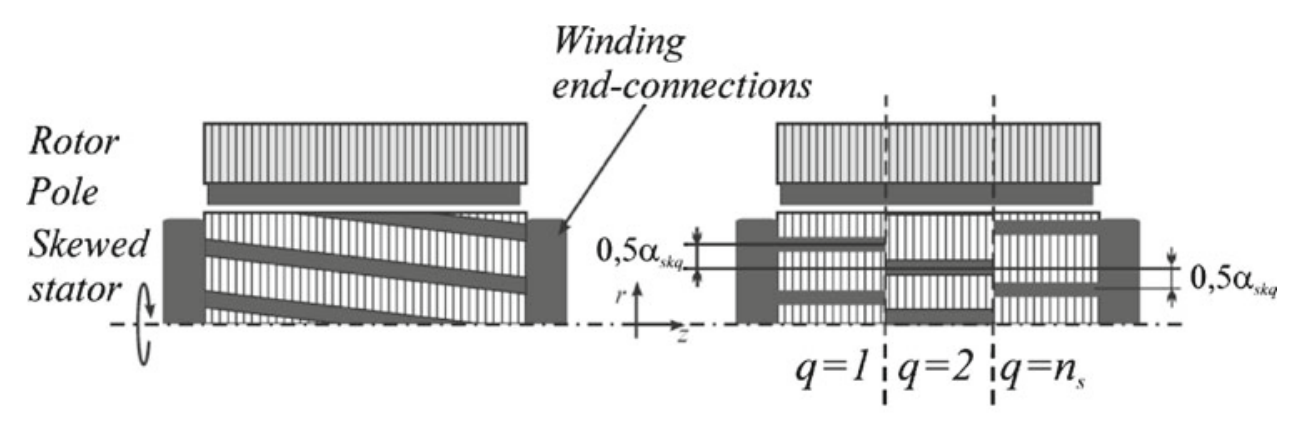

(a)

(b)

Fig. 3 Circuit model of a voltage waveinverter

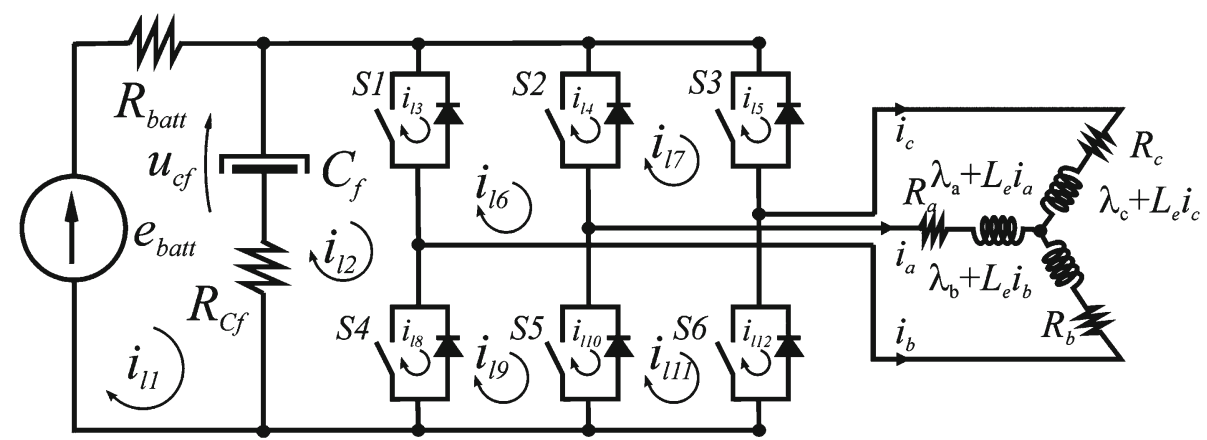

$\lambda_{u}=\frac{1}{n_{\mathrm{s}}} \sum_{q=1}^{n_{\mathrm{s}}}\left(\ell \sum_{n=1}^{2 p Q_{s}} \frac{n_{\mathrm{t}} \eta_{n}}{S_{u n}} \int_{S_{u n}} A \mathrm{~d} S\right)_{q}$

The Kirchhoff's equations of the circuit in Fig. 3 in matrix form are

$$
\begin{array}{r}
\boldsymbol{v}_{b}=\boldsymbol{R i}_{b}+\boldsymbol{L} \frac{\mathrm{d} \boldsymbol{i}_{b}}{\mathrm{dt}}+\boldsymbol{k}_{1} \frac{\mathrm{d}}{\mathrm{dt}} \boldsymbol{\lambda}+\boldsymbol{k}_{2} u_{c f} \\
0=C_{f} \frac{\mathrm{d} u_{C f}}{\mathrm{dt}}+\boldsymbol{k}_{2}^{T} \boldsymbol{i}_{b}
\end{array}
$$

Matrix $\boldsymbol{k}_{1}$ links phase linkage fluxes and vector $\boldsymbol{k}_{2}$ links the capacitor voltage with appropriate branches of the electric circuit. Using a standard approximation for magnetic vector potential i.e. $A \approx \sum_{i=1}^{3} N_{i} \varphi_{i}$, first-order triangular elements and the Galerkin weighted residual approach [13-15] equation (6) is written in the discrete form:

$$
\begin{aligned}
& v \int_{\Omega_{q}}\left(\nabla N_{i} \cdot \nabla N_{j}\right) \varphi_{i} \mathrm{~d} \Omega=\sum_{u} \sum_{n=1}^{2 p Q_{s}} \frac{n_{t} \eta_{u} \eta_{n} i_{u}}{S_{n}} \\
& \times \int_{\Omega_{q}} N_{i} \mathrm{~d} \Omega+\cdots+\int_{\Omega_{q}}\left(H_{c x} \frac{\partial N_{i}}{\partial y}-H_{c y} \frac{\partial N_{i}}{\partial x}\right) \mathrm{d} S
\end{aligned}
$$

Consequently, the vector of phase linkage fluxes is written as:

$\lambda_{u}=\sum_{q=1}^{N_{\mathrm{s}}}\left(\frac{\ell n_{\mathrm{t}}}{n_{\mathrm{s}}} \sum_{n=1}^{2 p Q_{s}} \frac{\eta_{n}}{S_{n}} \int_{S_{n}} \sum_{i=1}^{3} N_{i} \varphi_{i} \mathrm{~d} S\right)_{q}$

or in the more general form:

$\lambda=D \varphi$,

where $\boldsymbol{D}=\left[\boldsymbol{D}_{1}, \boldsymbol{D}_{2}, \boldsymbol{D}_{q}, \ldots, \boldsymbol{D}_{n_{\mathrm{s}}}\right], \boldsymbol{\varphi}=\left[\boldsymbol{\varphi}_{1}, \boldsymbol{\varphi}_{2}, \boldsymbol{\varphi}_{q}, \ldots\right.$, $\left.\boldsymbol{\varphi}_{n_{\mathrm{s}}}\right]^{T}$ and $D_{q i j}=\delta_{j} \frac{\ell n_{t} \eta_{n} \eta_{u}}{S_{n} N_{\mathrm{s}}} \int_{\Omega_{q}} N_{i} d \Omega, \delta_{j}= \begin{cases}0 & \text { if } \eta_{u} \neq 0 \\ 1 & \text { otherwise }\end{cases}$ Using loop analysis method for an electric circuit in Fig. 3, and considering (9) and (12), one obtains:

$\boldsymbol{k}_{l}^{T} \boldsymbol{u}_{s}=\boldsymbol{k}_{l}^{T} \boldsymbol{R} \boldsymbol{k}_{l} i_{l}+\boldsymbol{k}_{l}^{T} \boldsymbol{L} \boldsymbol{k}_{l} \frac{\mathrm{d} \boldsymbol{i}_{l}}{\mathrm{~d} t}+\boldsymbol{k}_{l}^{T} \boldsymbol{k}_{1} \frac{\mathrm{d}}{\mathrm{d} t} \boldsymbol{D} \boldsymbol{\varphi}+\boldsymbol{k}_{l}^{T} \boldsymbol{k}_{2} u_{c f}$

$0=C_{f} \frac{\mathrm{d} u_{c f}}{\mathrm{~d} t}+\boldsymbol{k}_{2}^{T} \boldsymbol{k}_{l} \boldsymbol{i}_{l}$,

where $\boldsymbol{k}_{l}$ is a loop matrix which transforms the circuit branch currents into a vector of loop currents $\boldsymbol{i}_{l}$. Using backward Euler formula [13] for approximation of time-derivatives in (12) results in the time-stepping system of field and circuit equations: 


$$
\begin{aligned}
& {\left[\begin{array}{ccc}
\boldsymbol{S} & -\boldsymbol{D}^{T} \boldsymbol{k}_{1}^{T} \boldsymbol{k}_{l}^{T} \ell^{-1} & \boldsymbol{0} \\
\frac{\boldsymbol{k}_{l}^{T} \boldsymbol{k}_{1} \boldsymbol{D}}{\Delta t} \boldsymbol{k}_{l}^{T}\left(\boldsymbol{R}+\frac{1}{\Delta t} \boldsymbol{L}\right) \boldsymbol{k}_{l} & \boldsymbol{k}_{l}^{T} \boldsymbol{k}_{2} \\
0 & \boldsymbol{k}_{2}^{T} \boldsymbol{k}_{l} & \frac{C_{f}}{\Delta t}
\end{array}\right]_{t}\left[\begin{array}{c}
\boldsymbol{\varphi} \\
\boldsymbol{i}_{l} \\
u_{C f}
\end{array}\right]_{t}} \\
& =\left[\begin{array}{c}
\boldsymbol{\Theta} \\
\boldsymbol{k}_{l}^{T} \boldsymbol{v}_{b}-\boldsymbol{k}_{l}^{T}\left(\boldsymbol{R}-\frac{1}{\Delta t} \boldsymbol{L}\right) \boldsymbol{k}_{l} \boldsymbol{i}_{l} \\
\frac{C_{f}}{\Delta t} u_{C f}
\end{array}\right]_{t-\Delta t},
\end{aligned}
$$

where $\boldsymbol{S}=\operatorname{diag}\left[\boldsymbol{S}_{1}, \boldsymbol{S}_{2}, \boldsymbol{S}_{q}, \ldots, \boldsymbol{S}_{n_{\mathrm{s}}}\right], \Theta=\left[\boldsymbol{\Theta}_{1}, \boldsymbol{\Theta}_{2}, \boldsymbol{\Theta}_{q} \ldots\right.$, $\left.\boldsymbol{\Theta}_{n_{\mathrm{s}}}\right]^{T}, S_{q i j}=v \int_{\Omega_{q}} \nabla N_{i} \cdot \nabla N_{j} d \Omega, \Theta_{q i}=\int_{\Omega_{q}}\left(H_{c x} \frac{\partial N_{i}}{\partial y}-\right.$ $\left.H_{c y} \frac{\partial N_{i}}{\partial x}\right) d \Omega$.

The transistor switches $S 1-S 6$ are modeled as two-state resistance elements, whilst the diodes as nonlinear voltagedependent current sources considering rudimentary static characteristics. In this way, the model allows to estimate overall efficiency of the drive including inverter power loss.

The electromagnetic torque of a motor is calculated from the Maxwell stress tensor. The modeling of rotor movement by means of elements distortion and permutation of node numbers in the machine air-gap allows for computation of time variations of phase currents, voltages and torque. Such a model provides a transient solution. If he constant rotor speed is assumed, the steady-state solution is obtained after a few periods of phase current from the beginning of computations. From the steady-state waveforms the rms and average values can be computed to provide information on power balance and losses.

\section{Analysis and results}

\subsection{Validation of the model}

The model was subdivided into seven mesh-slices in axial (z) direction. By several numerical experiments it has been confirmed that such an approximation of skew is sufficiently accurate from computational point of view. The distribution of magnetic flux over whole machine in steady-state no-load operation is shown in Fig. 4.

Figure 5a-d compares waveforms of phase current and phase-to-phase voltage at full-load conditions. The measured torque at the same point of operation was equal to $8.85 \mathrm{Nm}$, and the computed average torque was equal to $9.4 \mathrm{Nm}$, which considering the undetermined mechanical power loss, is very close to measurements. An agreement between the computed values of phase current, voltage and torque confirms the adequacy of the elaborated numerical model.

To support the theoretical consideration on the choice of skew angle based on (4) instead of (5), an additional calculation was carried out. A two-dimensional (space and time)

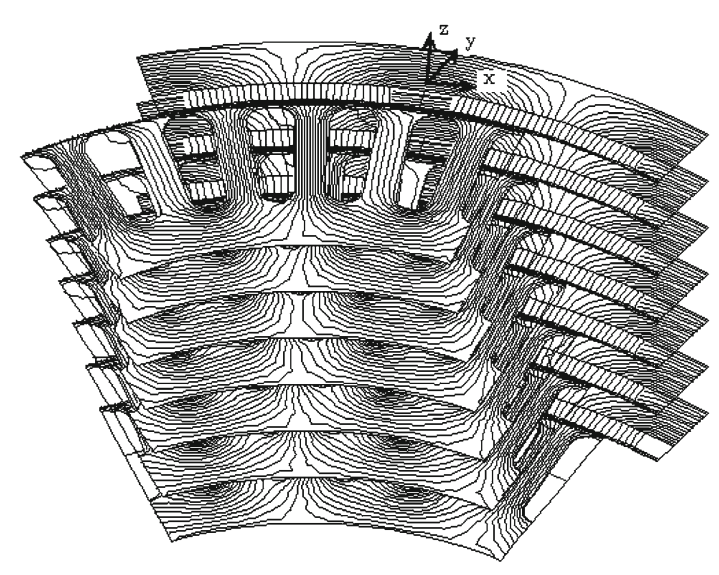

Fig. 4 Magnetic flux distribution over multi mesh-slice motor model under no-load conditions considering symmetry with respect to one pole-pair

Fourier decomposition for radial $\left(B_{\mathrm{r}}\right)$ and tangential $\left(B_{\psi}\right)$ component of an air-gap magnetic flux density is carried out as the rotor rotates at no-load. The results limited to first 20 harmonics are shown in Fig. 6.

In the analysis the three lot-fill factors are considered. A first case (Fig. 6a, b) refers to the machine with specifications given in Table 2, with the slot fill factor equal to 0.778 . The two remaining cases are referred to the machines having slot-fill factors equal to 0.6 and 0.9 (see Fig. 6c, d and Fig 6e, $\mathrm{f}$, respectively). Owing to the fact that the torque is in proportion to a product $B_{\mathrm{r}} B_{\psi}$, it can be seen in all figures that the most influential is harmonic $Q / p+1$ (equal here to 7) with no matter what the slot-fill factor is. This confirms that Eq. (4) is the best choice to calculate the skew angle for such a motor.

\subsection{Operation at no-load conditions}

As it can be observed in Fig. 1b, c, the stator sheet stack of the physical motor is skewed by one slot pitch. From the analysis carried out in the introductory section, it is clear that a relative skew for the considered motor is 0.857 of slot-pitch width (see Table 1). To examine the effect of this change, the calculations were performed for different cases. From the results of calculations, which are exposed in Fig. 7, it can be noticed that skewing the stator by angle calculated from (4) reduces cogging torque much better than skewing it by one slot-pitch. The cogging torque reduction is nearly total.

The skew by one slot pitch gives the value of fundamental skew factor of 0.955 , whilst the second option gives $k_{s k 1}$ equal to 0.967 . In the considered motor, the fundamental winding factor is equal to the skew factor due to unity fundamental coil-span and distribution factors.

Figure 8 compares computed waveforms of phase back emf. A smaller skew angle results in more trapezoidal waveforms of back emf rather than in meaningful rise in 


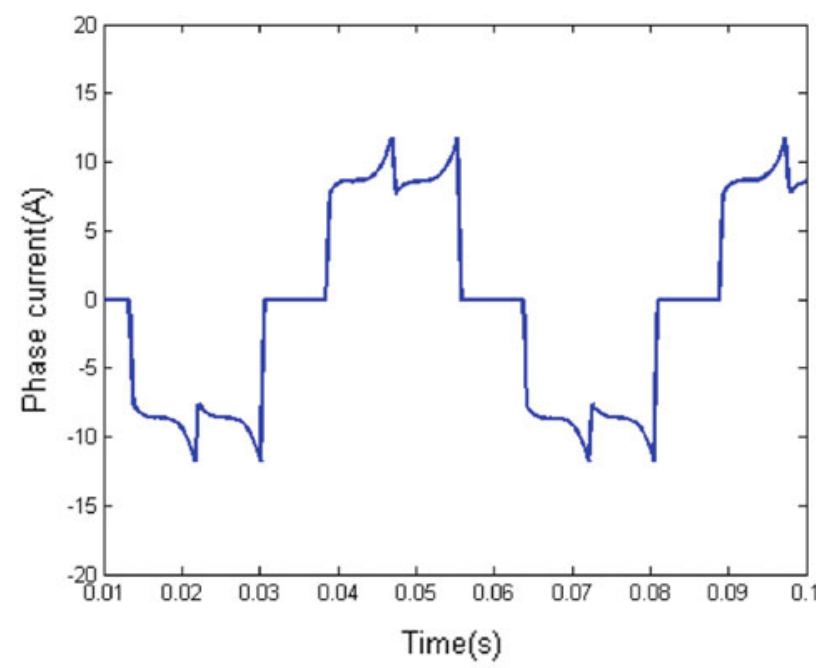

(a)

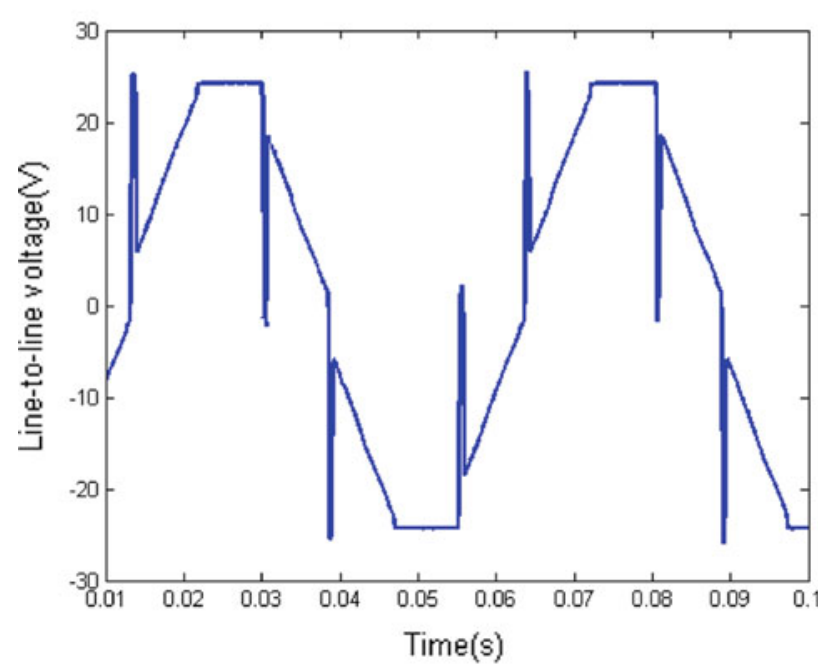

(c)

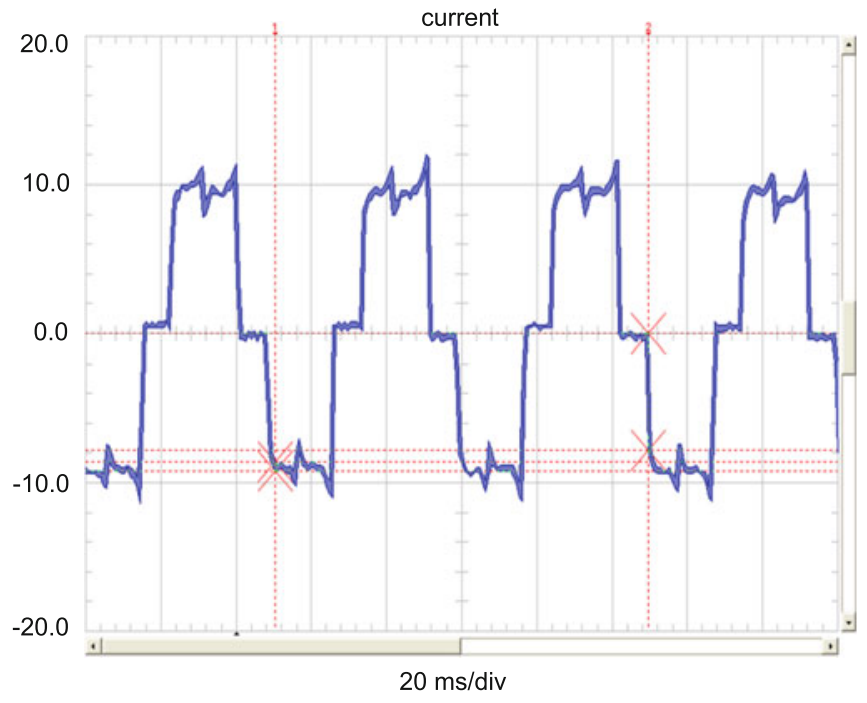

(b)

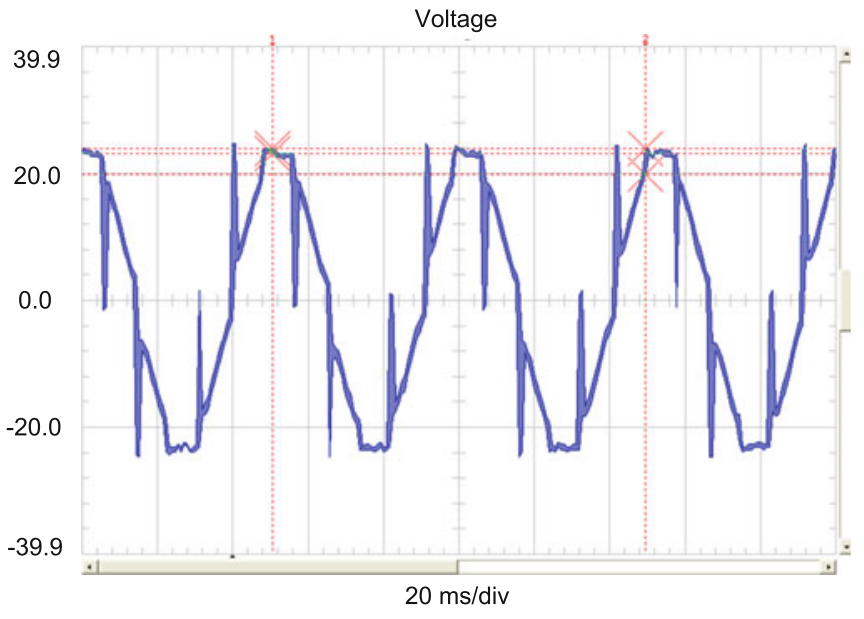

(d)

Fig. 5 Waveforms of phase current $(\mathbf{a}, \mathbf{b})$, and line-to-line voltages $(\mathbf{c}, \mathbf{d})$ at rotor speed of $170.4 \mathrm{rpm}$ and supply voltage of $24.4 \mathrm{~V}$ : computations $(\mathbf{a}, \mathbf{c})$, measurement results $(\mathbf{b}, \mathbf{d})$

magnitude. Because a reduction of the cogging torque is very high, it will be potentially useful to expand the width of magnet pole to full pole-pitch. This makes the waveform of phase back emf better, nearly trapezoidal shape.

Expansion of the pole width in the latter to the length of the pole-pitch results in drop of efficiency from 82 to $81.1 \%$ (see Fig. 9), which is due to higher value of the core loss. As it was already mentioned, the above values of motor efficiency were calculated taking into account the inverter power loss. Variations of instantaneous core loss for the same conditions of operation are shown in Fig. 10. Averaging the waveforms in time gives the value of $1.92 \mathrm{~W}$ for the motor without a skewed stator and, respectively, 1.66 and $1.65 \mathrm{~W}$ for the two remaining motor versions.
Expansion of pole width causes a rise in core loss to $1.90 \mathrm{~W}$, which is due to the higher rms value of magnetic flux density in the motor air-gap as compared to other motor versions. Also from this point of view, it is better to keep the ratio of pole width to the pole-pitch at a typical value of approximately 0.8 rather than increase it to unity. An obvious effect of skewing is a reduced pulsation of the electromagnetic torque. From the results of instantaneous electromagnetic torque computations plotted in Fig. 11, it is clear that the skew angle calculated from Eq. (4) provides the smallest torque pulsations which are practically due only to commutation effects.

A side effect of skewing is a reduction of the magnitude of axial component of current density by the factor of $\sec \alpha_{s k}$ 


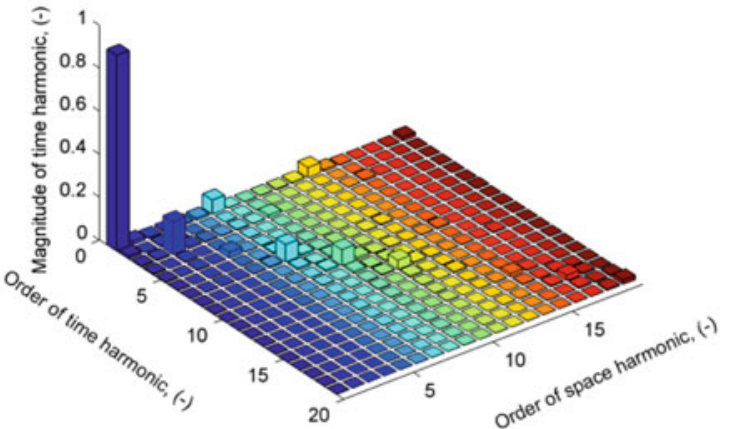

(a)

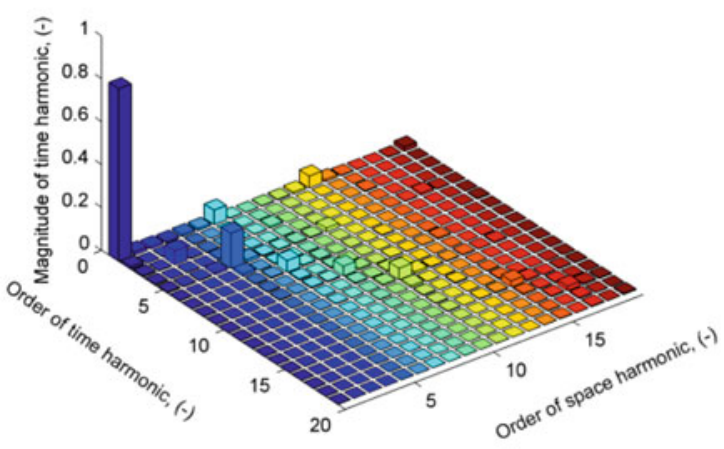

(c)

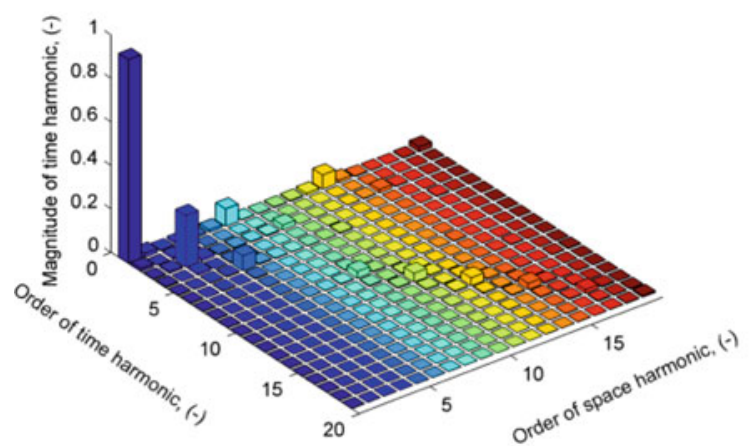

(e)

Fig. 6 Two-dimensional harmonic spectra of components of an airgap flux density: a, b normal and tangential component for pole width-to-pole-pitch equal to $0.778, \mathbf{c}, \mathbf{d}$ normal and tangential compo-

which can be considered favorable in applications, where the demagnetization effects are particularly important.

\section{Conclusions}

Theoretical considerations and computations using a quasi3D multi mesh-slice finite element model show that the best angle of skew of the sheet pack for the three-phase integer-slot permanent magnet machine with the surfacemounted magnets can be determined from a simple analytic formula. Application of a skew angle determined in such a

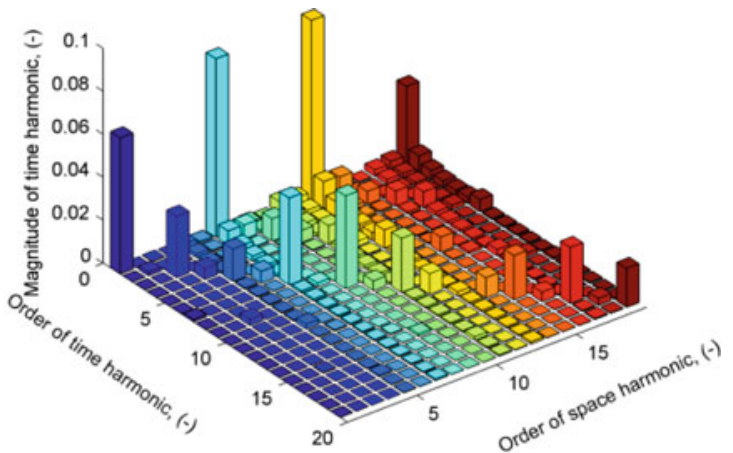

(b)

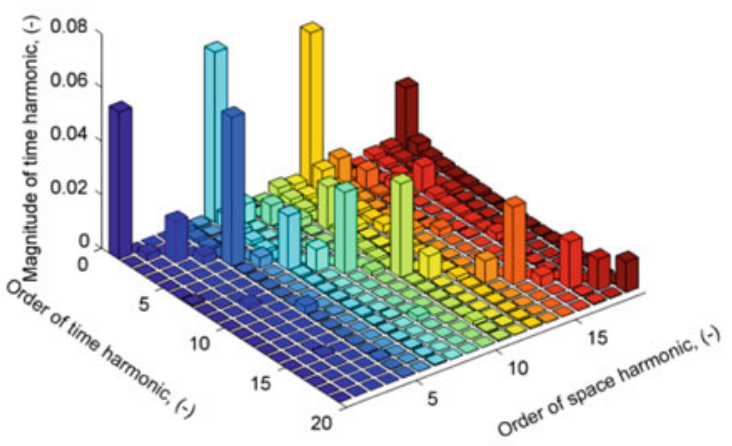

(d)

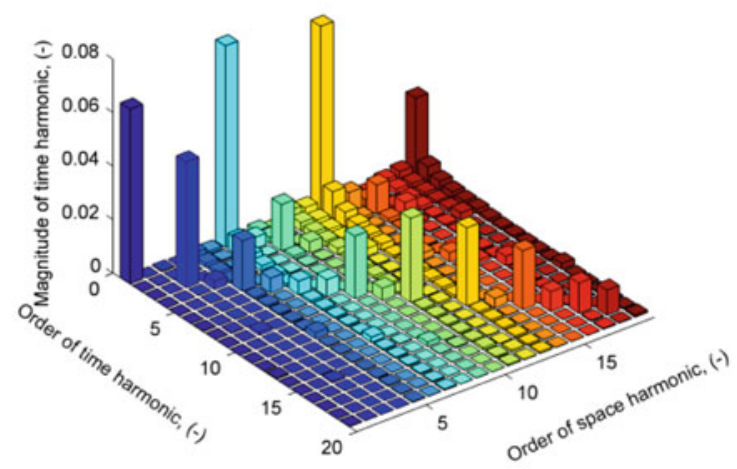

(f)

nent for pole width-to-pole-pitch equal to $0.6, \mathbf{e}, \mathbf{f}$ normal and tangential component for pole width-to-pole-pitch equal to 0.9

way provides a very high reduction of electromagnetic torque pulsations caused by the angular variation of the equivalent magnetic reluctance of an air-gap.

The results of analysis have led to a few useful conclusions, but it is worth noting that these are based merely on a numerical experiment. The possibility of accomplishment of such an effect in a real machine is the matter of accuracy in the manufacturing process. This is because sensitivity of the magnitude of the cogging torque to the variation of the skew angle is very high.

Because an amplitude of the fundamental harmonic of the cogging torque is in proportion to the product of magnitudes 


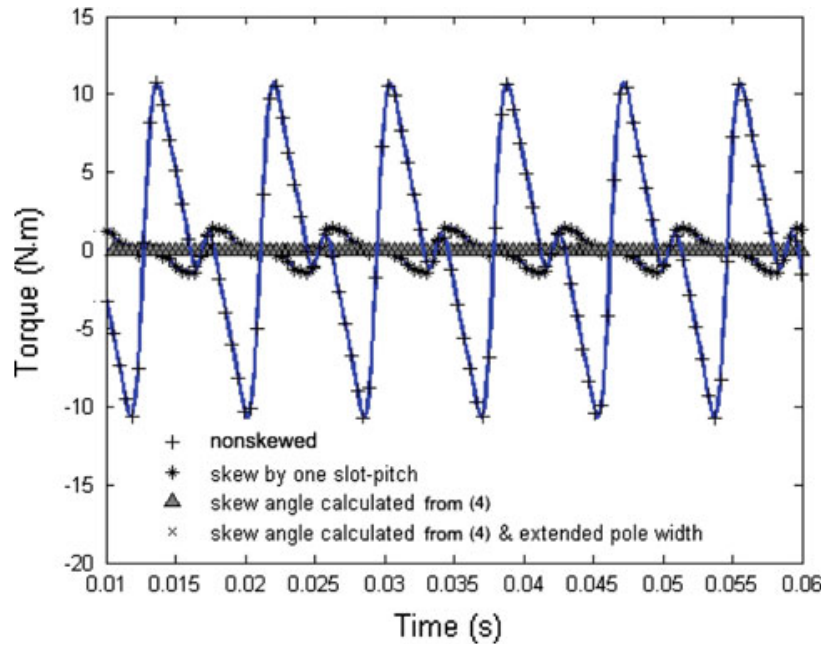

Fig. 7 Cogging torque calculated for two different skew angles and for the motor without skew

of the two most important harmonics of magnetic flux density, which were identified in the preceding sections, it is possible to estimate the amplitudes of the cogging torque in skewed motor $\left(T_{e s k}\right)$ related to that in the motor without a skew $\left(T_{\text {ens }}\right)$ as:

$\frac{T_{\text {esk }}}{T_{\text {ens }}} \geq k_{s k 1} k_{s k}(\operatorname{lcm}(2, Q / p)+1)$

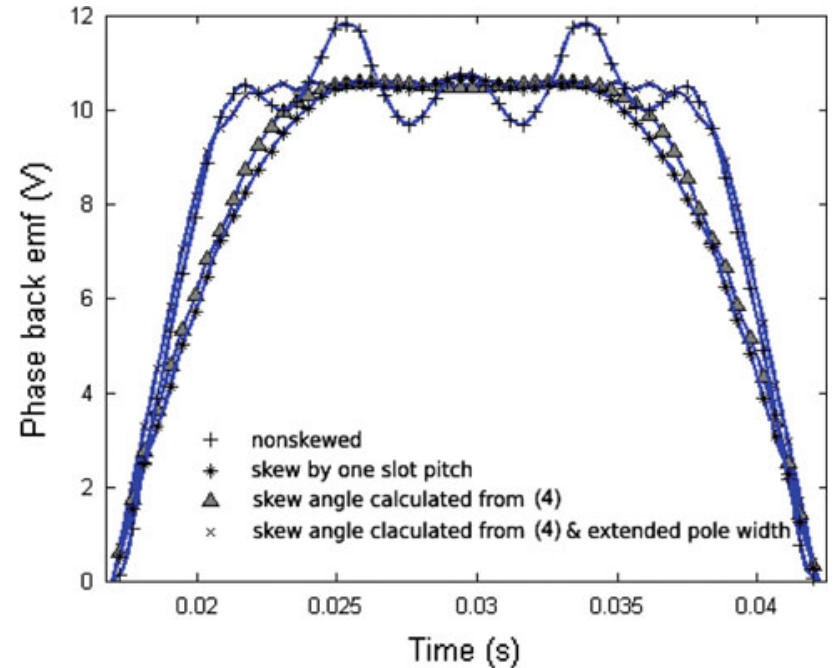

Fig. 8 Waveforms of phase back emf calculated for two different skew angles and for motor without skew at rotational speed of $170.4 \mathrm{rpm}$

Using the specifications of the machine considered and (15), it can be shown that discrepancy in a skew angle by $5 \%$ from value given by (4) is responsible for the cogging torque magnitude, which is at least $16 \%$ of that in the motor without a skew. This prediction agrees with the results presented in Fig. 7.

The results presented were obtained for a fractional power machine due to limitations of laboratory facilities but these
Fig. 9 Efficiency (a), and core loss (b) versus output power at supply voltage of $24.4 \mathrm{~V}$ (a)

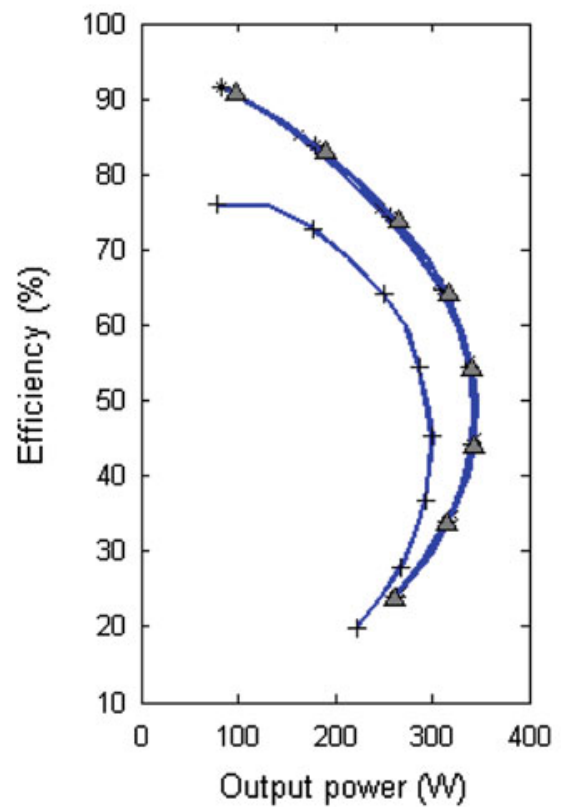

+ nonskewed

* skew by one slot-pitch

$\triangle \quad$ skew angle calculated form (4)

$\times \quad$ skew angle calculated form (4) \& extended pole width (b)

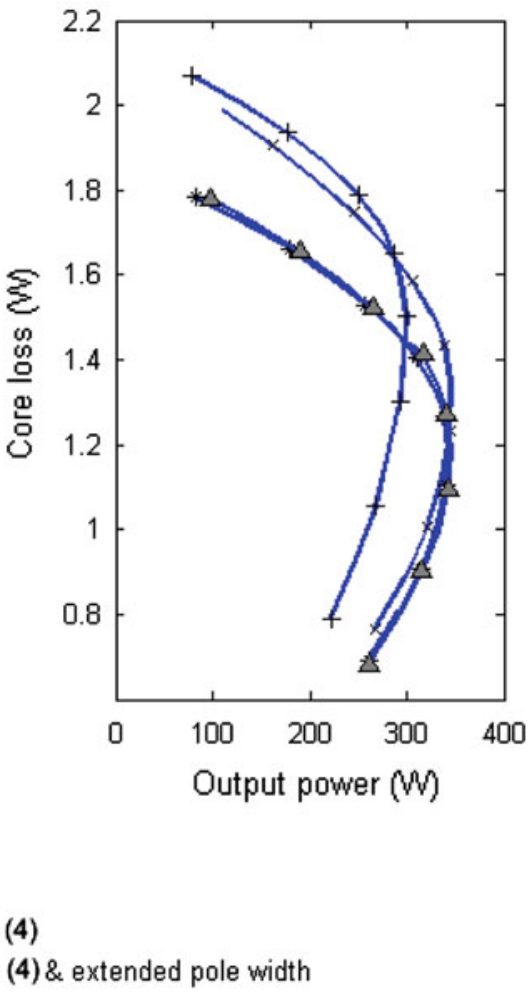




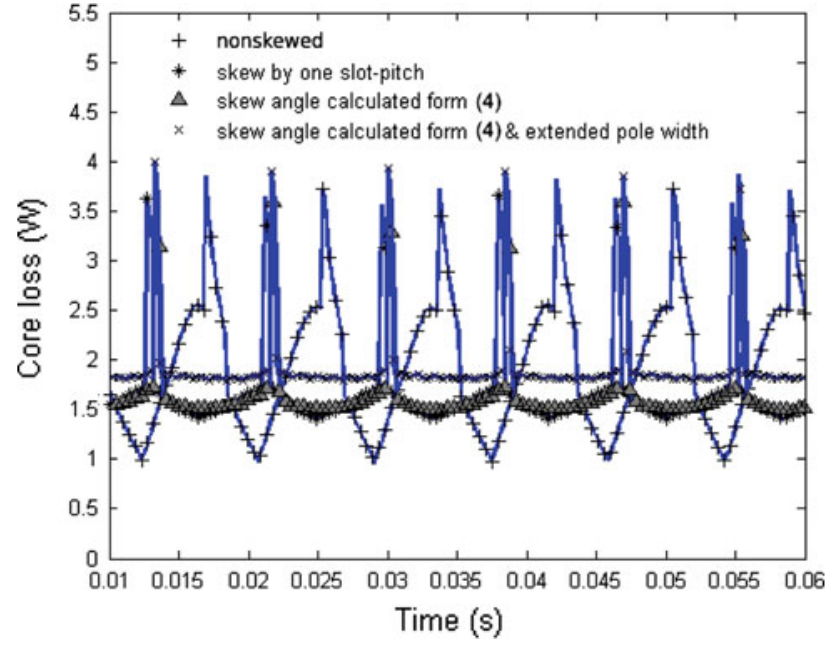

Fig. 10 Variations of core loss versus time for different skew angles at rotor speed of $170.4 \mathrm{rpm}$ and supply voltage of $24.4 \mathrm{~V}$

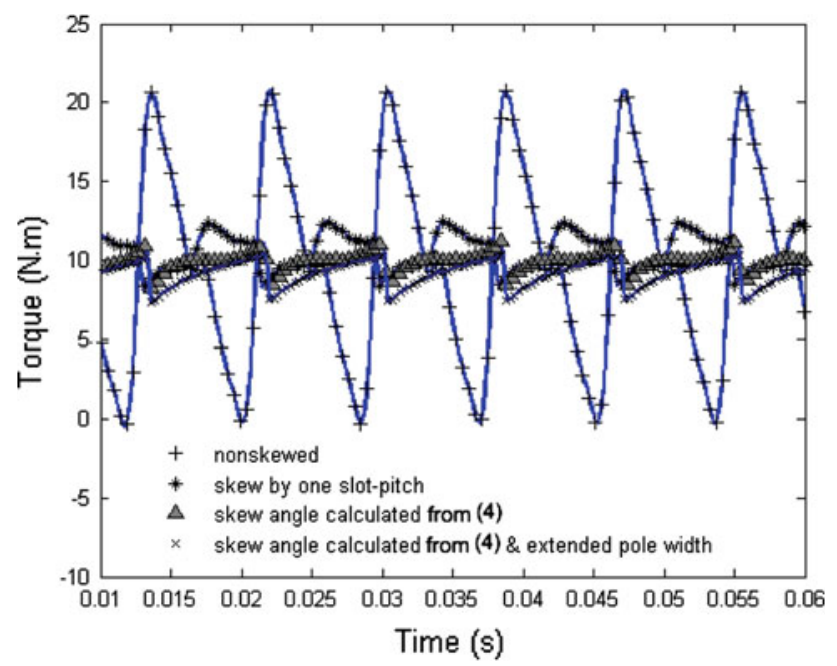

Fig. 11 Variations of electromagnetic torque vs. time for different skew options at constant rotor speed of $170.4 \mathrm{rpm}$ and supply voltage of $24.4 \mathrm{~V}$

should be addressed particularly to larger machines where the problem of reduction of core loss and mechanical vibrations is crucial.

Acknowledgments This work was supported by the National Science Foundation under the Grant ECCS-0801671.

Open Access This article is distributed under the terms of the Creative Commons Attribution License which permits any use, distribution, and reproduction in any medium, provided the original author(s) and the source are credited.

\section{Appendix}

The model for estimation of instantaneous lamination core loss was developed considering as a basis classical Bertotti equation $[16,17]$ that describes the core loss density per unity mass as:

$\Delta \bar{p}_{f e}=\frac{\pi^{2} d^{2} \sigma}{6 \rho}|B|^{2} f^{2}+c_{\mathrm{h}}|B|^{2} f+c_{\mathrm{e}}|B|^{\frac{3}{2}} f^{\frac{3}{2}}$

This equation is defined in the domain of frequency. The total core loss is determined by integrating (16) over motor mass. Coefficients $c_{h}$ and $c_{e}$ are determined by means of a standard procedure which relies upon performing linear regression based on loss curves provided by manufacturers of magnetic materials. For the material considered in this work, $d=0.35 \mathrm{~mm}, \sigma=1.92 \times 10^{6} \mathrm{~S} / \mathrm{m}, \rho=7,65 \mathrm{~kg} / \mathrm{m}^{3}, c_{\mathrm{h}}=$ $0.02004, c_{\mathrm{e}}=0.00015$. In order to transform (16) from frequency to time domain the frequency is replaced with:

$f \equiv|B|^{-1}\left|\frac{\mathrm{d} B}{\mathrm{~d} t}\right|$

Using a sinusoidal variation of magnetic flux density as a test waveform, and equating all components of a newly obtained time-domain equation, one obtains the instantaneous core loss density per a mass unit:

$\Delta \bar{p}(t)=\frac{\mathrm{d}^{2} \sigma}{12 \rho}\left|\frac{\mathrm{d} B}{\mathrm{~d} t}\right|^{2}+c_{\mathrm{h}}^{*}\left|\frac{\mathrm{d} B}{\mathrm{~d} t}\right||B|+c_{\mathrm{e}}^{*}\left|\frac{\mathrm{d} B}{\mathrm{~d} t}\right|^{\frac{3}{2}}$,

where $c_{\mathrm{h}}^{*}=0.25 \cdot c_{\mathrm{h}}, c_{\mathrm{e}}^{*}=0.11 \cdot c_{\mathrm{e}}$. Considering the twodimensional distribution of magnetic field in the motor model over $q$ th mesh-slice, (18) takes the form:

$$
\begin{array}{r}
\Delta \bar{p}_{q}(t)=\frac{\mathrm{d}^{2} \sigma}{12 \rho}\left(\left(\frac{\mathrm{d} B_{u}}{\mathrm{~d} t}\right)^{2}+\left(\frac{\mathrm{d} B_{v}}{\mathrm{~d} t}\right)^{2}\right)_{q}+\cdots \\
+c_{\mathrm{h}}^{*}\left(\left|\frac{\mathrm{d} B_{u}}{\mathrm{~d} t}\right|\left|B_{u}\right|+\left|\frac{\mathrm{d} B_{v}}{\mathrm{~d} t}\right|\left|B_{v}\right|\right)_{q} \\
+c_{\mathrm{e}}^{*}\left(\left(\frac{\mathrm{d} B_{u}}{\mathrm{~d} t}\right)^{2}+\left(\frac{\mathrm{d} B_{v}}{\mathrm{dt}}\right)^{2}\right)_{q}^{\frac{3}{4}},
\end{array}
$$

where coordinates $u$ and $v$ stand for $x$ and $y$ in the stator region and $r$ and $\psi$ in the rotor region.

The total core loss is obtained from core loss formula (18). It describes a variation of core loss density in time. To obtain power loss at given operation conditions, it must be integrated over the motor core volume and averaged in time:

$$
\Delta P_{f e}=\sum_{q=1}^{n_{\mathrm{s}}} \int_{\Omega_{q}} \int\left(\frac{\rho \ell}{T n_{\mathrm{s}}} \int_{0}^{T} \Delta \bar{p}_{q}(t) \mathrm{d} t\right) \mathrm{d} S
$$

\section{References}

1. De La Ree H, Boules N (1989) Torque production in permanentmagnet synchronous motors. IEEE Trans Ind Appl 25:107-112

2. Ishikawa T, Slemon GR (1993) A method of reducing ripple torque in permanent magnet motors without skewing. IEEE Trans Magn 29:2028-2031 
3. Hendershot JR, Miller TJE (1994) Design of brushless permanentmagnet motors. Magna Physics Publishing, Clarendon Press, Oxford

4. Jahns TM, Soong WL (1996) Pulsating torque minimization techniques for permanent magnet $\mathrm{AC}$ motor drives-a review. IEEE Trans Ind Electr 43(2):321-330

5. Hwang S, Lieu DK (1994) Design technique for reduction of reluctance torque in brushless permanent magnet motors. IEEE Trans Magn 30:4287-4289

6. Chen SX, Low TS, Bruhl B (1998) The robust design approach for reducing cogging torque in permanent magnet motors. IEEE Trans Magn 34(4):2135-2137

7. Salon S, Sivasubramaniam K, Tukenmez E (2000) An approach for determining the source of torque harmonics in brushless DC motors. In: Proceedings of 2000 international conference on electrical machines ICEM'00, pp 1707-1711

8. Łukaniszyn M, Jagiela M, Wrobel R, Latawiec K (2004) 2D Harmonic analysis of the cogging torque in synchronous permanent magnet machines. Int J Comput Math Electr Electron Eng COMPEL 23(3):774-782

9. Lukaniszyn M, Jagiela M, Wrobel R (2004) Optimization of permanent magnet shape for minimum cogging torque using a genetic algorithm. IEEE Trans Magn 40(2):1228-1231

10. Magnussen F, Sadarangami C (2003) Winding factors and Joule losses of permanent magnet machines with concentrated windings. In: Proceedings of 2003 international electric machines and drives conference IEMDC'2003, vol 1, pp 333-339
11. Skaar SE, Krovel O, Nilssen R (2006) Distribution, coil-span and winding factors for PM machines with concentrated windings. In: Proceedings of 2006 international conference on electrical machines ICEM'06, Incl. on Conf CD, Paper No 346

12. Gyselinck JJC, Vandelvelde L, Melkebeek JAA (2001) Multi-slice FE modeling of electrical machines with skewed slots-the skew discretization error. IEEE Trans Magn 37:3233-3237

13. Zienkiewicz OC, Taylor RL (2000) Finite element method, 5th edn. Butterworth-Heinemann, Oxford

14. Ho SL, Fu WN, Li HL, Wong HC, Tan H (2001) Performance analysis of brushless DC motors including features of the control loop in the finite element modeling. IEEE Trans Magn 37(5):33703374

15. Jabbar MA, Liu Z, Dong J (2003) Time-stepping finite-element analysis for the dynamic performance of a permanent magnet synchronous motor. IEEE Trans Magn 39(5):2621-2623

16. Bertotti G (1988) General properties of power losses in soft ferromagnetic materials. IEEE Trans Magn 24(1):621-630

17. Lin D, Zhou P, Fu WN, Badics Z, Cendes ZJ (2004) Dynamic core loss model for soft ferromagnetic and power ferrites materials in transient finite element analysis. IEEE Trans Magn 40(2):1318 1321 